\title{
Effective screening in child health
}

\author{
Give deaf children a better deal and look more broadly at other problems
}

I $\mathrm{n}$ Britain today two neonatal screening programmes-for phenylketonuria and congenital hypothyroidism-are working well and preventing brain damage and severe intellectual impairment from these conditions. But for many of the other child health problems discussed last month at a meeting on systematic reviews of screening in child health organised by the National Screening Committee and the Royal College of Paediatrics and Child Health there are doubts either about the value of early detection or about the most effective method for doing it.

Each year in the United Kingdom about 700 children are born with severely impaired hearing, and identifying them is important because early treatment improves their language development and emotional well being. The traditional distraction test of hearing at 8 months is failing them: the mean age for detecting severe deafness is 18 months. The evidence reviewed at the meeting shows clearly that universal (rather than targeted) electronic screening of newborn infants is more reliable, cheaper, and leads to hearing aids being fitted at a mean age below 4 months.

For other conditions future policy looks less clear. The list of inborn errors of metabolism which could be detected in the newborn has greatly expanded: all are rare, and some are treatable, with the possibility of avoiding brain damage. Only two-glutaric aciduria type 1 and medium chain acyl coenzyme A dehydrogenase deficiency-fulfil the conditions which justify screening, which has to be done by tandem mass spectrometry. Tandem mass spectrometry (which could also detect several rarer disorders) would need to be concentrated in about six centres in Britain and could be cost effective only if it was also used for the phenylketonuria screenbut this means replacing a system which is in place and working well. A pilot scheme, based on one laboratory serving a population of a few million, was suggested as a possible way forward.

Two long established screening programmes are in scientific disarray, having been set up with inadequate evidence that they were detecting conditions that benefit from early treatment. Preschool vision testing was driven by a strong belief that there is a sensitive period for visual development and that amblyopia must be detected and treated early. There is no evidence for this from controlled trials in children, probably because the belief was so strong that such trials could not ethically be done. Intense disagreement exists about what to do now with a screening programme based on a scientifi- modern standards of clinical research. Lack of evidence of benefit is not evidence of no benefit, but some at the meeting argued that no screening programme which fails to meet current scientific criteria should be allowed to continue.

Screening for congenital dislocation of the hip is in similar confusion. The Ortolani or Barlow test for hip instability has been universally used in Britain for 30 years. It fails to identify two thirds of the hips which subsequently need surgical treatment and has made little or no difference to the number coming to surgery. It causes many infants to be splinted who probably have no hip disorder. Some countries now use ultrasound screening instead, but the most evident result is an increase in the number of children without the disease who are treated by splinting.

Cystic fibrosis and haemoglobinopathies are among the conditions where there are important questions about screening, but which were not discussed at the meeting. A far bigger issue was raised about two much commoner disorders. Speech and language delay is the commonest neurodevelopmental disorder, and behaviour problems are the commonest cause of disability in childhood. The screen and treat model, as applied to phenylketonuria, or even deafness, is unpromising for these problems, and Sarah Stewart-Brown (from the Health Services research Unit, Oxford) offered a moving vision of a different approach. In screening for, say, phenylketonuria, we are identifying a small group who differ qualitatively from the rest of the population. Children's behaviour problems, however, represent the unhappy end of a distribution curve. Rather than make an arbitrary cut off for who needs treatment, she argued, we should try to shift the whole distribution by helping the whole population. This requires indirect intervention, by mobilising the skills of parents and teachers. An encouraging amount of evidence exists that such indirect intervention benefits children with language delays and improves parenting skills and mother-child interactions. Health visiting is currently under threat in Britain as an expensive service of unproved value, yet the evidence produced at meeting argued strongly against both these criticisms, and home visiting would be a cornerstone of the structure for improving children's emotional well being which Stewart-Brown envisages. "Emotional literacy" teaching in schools has also been shown to enhance young people's self esteem and considerateness, with huge potential gains in social and health related behaviour. A cost effective 
policy for promoting child health must look both at screening for the rare disorders and at community interventions for the common ones. David Hall, professor of community paediatrics in Sheffield and convenor of the meeting, believes a body is needed with overall responsibility for public health in childhood; it would need to work jointly with the education service. Certainly the issues discussed at the meeting go well beyond the brief of the National Screening Committee. Meanwhile, we need a quick decision from the committee on newborn screening for deaf children, who deserve a better deal than they are getting, and a thoughtful one on tandem mass spectrometry screening for metabolic disorders, before local policies change in a haphazard way.

Roger Robinson Associate editor, BMJ

A full meeting report will be available from the National Screening Committee, c/o Child growth Foundation, 2 Mayfield Avenue, London W4 1PW (£15). A repeat session of the meeting will be held on 8 January 1998: fax 01819959075 for information.

\title{
Ultrasound for the diagnosis of deep vein thrombosis: where to now?
}

\author{
A new protocol for diagnosis and treatment
}

$\mathrm{I}$ $\mathrm{n}$ this issue Cogo and others from Italy, Canada, and the Netherlands describe using two ultrasound examinations a week apart in 1702 outpatients with suspected deep vein thrombosis of the leg to determine whom to treat (p 17). ${ }^{1}$ Four hundred and twelve were diagnosed with thrombosis by this procedure; the rest were observed without treatment for six months. Nine of the untreated patients developed proof of thromboembolic disease during follow up: two had pulmonary embolism, fatal in one. Overall, fewer than $1 \%$ of patients presenting with possible deep vein thrombosis were missed with the authors' approach and only one $(<0.1 \%$ of patients enrolled) paid with his life. Is the authors' protocol now ready for routine use? Is this the requiem for the venogram?

The protocol was straightforward. The authors used the least expensive type of ultrasound imaging: grey scale real time ultrasound. Doppler signals, manoeuvres to change venous flow, and colour were not used. While Doppler and colour technology are essential for measuring valvular regurgitation echocardiographically and certain other applications, they appear unnecessary in the authors' hands for identifying deep vein thrombosis requiring treatment.

The authors used full compressibility of the vein-that is, complete obliteration of the vein lumen as visualised on the ultrasound screen after pressure with the transducer probe-as the sole criterion for clot. They have previously validated this technique. ${ }^{2}$ They compressed each leg at just three sites: the common femoral vein next to the artery at the femoral ligament, the popliteal vein next to the artery behind the knee, and the same vein about eight centimetres distal, where it trifurcates into smaller calf veins. These manoeuvres can be easily and quickly learnt, although interpretation of the result at the distal popliteal vein is tricky. The first two sites are readily identifiable but inclusion of the third site was a mixed blessing: it allowed detection of more thromboses (thereby reducing the need for follow up studies from two to one ${ }^{3}$ ), but caused false positive readings, reducing the specificity of the result and the positive predictive value of clot detection at this distal site. Failure of compressibil- ity at the first two sites was proved to be thrombus by contrast venography $99 \%$ of the time but at the third site only $79 \%$ of the time. While some doctors may follow symptomatic distal popliteal vein clots for propagation before treating, many will anticoagulate. If these results are generalisable, about $20 \%$ of patients with abnormal compressibility limited to the distal popliteal vein will be falsely positive and unnecessarily subjected to anticoagulation. Fortunately, a compression ultrasound examination abnormal solely at this distal site was rare, occurring in only $23(1.4 \%)$ patients.

Only $12(3 \%)$ of the 412 patients with abnormal ultrasound examinations were picked up at the second test a week after presentation; by then, 400 patients with abnormal tests had already been identified. Is it worth bringing 1300 patients back to find thrombi in 12 (roughly 1\%)? We think it is. A follow up visit a week after deep vein thrombosis is suspected is reasonable, and the consequences of missing those 12 patients' disease are worrisome. The cost of each ultrasound examination should not be high, far less than that of venography or empirical anticoagulation.

The study results appear valid. Only $0.7 \%$ of patients studied slipped through the two ultrasound examinations to present with thrombosis or embolism. Even if we assume that the eight protocol violators (one patient with venous thrombosis found at elective venography and seven given anticoagulation without objective evidence of thrombosis) all truly had thrombosis and had been missed by the second examination, the failure rate of the protocol would still be only $1.4 \%$ over six months.

These results may not be generalisable to patients excluded from the study, especially pregnant patients and those with previous leg thromboses. Recurrent leg thromboses are not uncommon. ${ }^{45}$ Nevertheless, were low cost ultrasound devices, ${ }^{6}$ the limited expertise required for these simple examinations, and low molecular weight heparin for treatment ${ }^{7-10}$ all readily available to practitioners, this new paradigm for diagnosis and treatment would represent a profound change in care, to the advantage of many thousands of patients worldwide. Diagnosis would be simple and 